\title{
Reaction of water and living systems under the chronic effect of ionized radiation in low doses
}

\author{
Igor Borisovich Ushakov, Vladimir Vladimirovich Tsetlin, Svetlana Stepanovna Moisa
}

Federal State-Financed Establishment of Science, State Scientific Center of The Russian Federation, Institute of Biomedical Problems of The Russian Academy of Sciences, Moscow, Russia

Email: v_tsetlin@mail.ru

Received 23 October 2012; revised 28 November 2012; accepted 11 December 2012

\begin{abstract}
It is represented the review about the effect of low doses of ionized radiation on different types of biological objects (Japanese quail embryos, Aspergillus niger, Spirostomum ambiguum Ehrbg., mezenchim stem cells of mice bone brain, dry seeds of the highest plants, blood lymphocytes of pilots and cosmonauts) and water medium. In model experiments under the chronic ionized radiation in doses comparable with the doses of ionized radiation inside the orbital space stations and during the flight in interplanetary space was shown alike with morphological deviations (Japanese quail embryos, Aspergillus niger), the phenomenon of radiation hormezis (Aspergillus niger, mezenchim stem cells), the increasing of the germination of seeds, the decreasing of spontaneous motion activity of spirostoms and DNA damage, chromosome aberrations and the increased radio-sensitivity to adding radiation load in blood lymphocytes. These data testified the fact that the definite factor of ionized radiation effect is the changing of water medium state. Thus under the interplanetary cosmic flight and long stay on the orbit in the region of magnetosphere the studying kinds of radiation first effected on the water medium of organism as a result morpho-functional structures were changed.
\end{abstract}

Keywords: Ionized Radiation; Reaction of Water; Living Systems; Low Doses; Orbital Space Station

\section{INTRODUCTION}

The effect of ionized radiation in doses within $30-50$ cGy is studied on the different biological objects [1-5]. At the same time the effect of ionized radiation on living organism in more yet low doses not exceeding $10 \mathrm{cGy}$ is remained to be weak-studying. In addition to the question about the correlation of affects on organism of the immediate effect of ionized emanation and the influence of changing environment due to the radiation irradiation [6]. Literature data about the influence of different doses of ionized radiation on the living organisms are not one-digit and even contradictorily [7]. The influence of low doses $(<1-10 \mathrm{cGy})$ is studied not enough, in this connection the common regularities of its effects on biological objects are unknown. For the more deep understanding of the mechanism of indirect influence of radiation, in our opinion, it should pay attention to the explorations of some physic properties of the inner and outer water medium, exposing by low intensive irradiation of ionized emanation of different origin and its effect on biological objects [6]. As it is known the ionized radiation influences on the cell functions of all levels of organization. There are some different types of ionized radiation effects in low doses on living organisms represented in Table 1.

\section{ASPERGILLUS NIGER}

It is established earlier that the variations of radiation conditions modulated the processes of stagnation and activation of microbial association on the orbital station "MIR" [6,8]. For this in conditions of maximal power of absorb dose $600-800 \mathrm{mkGy} /$ day and minimum of solar activity (SA) colony-formed units (CFU) micromycetes and bacterium could increase by a factor of ten thousand and represent the real danger for the crew and station equipment [6]. The effect of radiation conditions in compartments of orbital station "MIR" showed in uneven dynamics of the microbial association development settling the surface of interior and equipment. It is established the existence of the correlation between the alterations of numbers of CFU of micromycetes and bacterium and the parameters of SA [9]. Under the station flight in the period of minimum SA marked the increasing of numbers of viable fragments of microorganisms to $10^{6} \mathrm{CFU}$ per $100 \mathrm{sm}^{2}$ of surface (for the average value $10^{3} \mathrm{CFU}$ ). More that in the period of the most power of absorb dose (PAD) on the ship's side of orbital complex 
Table 1. The effect of the different types of ionized radiation in low doses on biological objects.

\begin{tabular}{|c|c|c|c|c|c|c|c|c|}
\hline \multirow[b]{2}{*}{ Type of exposure } & \multicolumn{8}{|c|}{ Type of biological object } \\
\hline & $\begin{array}{l}\text { Asperg. } \\
\text { niger }\end{array}$ & Spiro-stoms & $\begin{array}{l}\text { Dry } \\
\text { seeds }\end{array}$ & $\begin{array}{c}\text { Bone } \\
\text { brain } \\
\text { mezen } \\
\text { chim stem } \\
\text { cells }\end{array}$ & $\begin{array}{l}\text { Bone } \\
\text { brain } \\
\text { stroma }\end{array}$ & $\begin{array}{l}\text { Hemato-poetic } \\
\text { ancestor cells }\end{array}$ & $\begin{array}{l}\text { Human } \\
\text { Lympho } \\
\text { cytes }\end{array}$ & $\begin{array}{l}\text { Quail } \\
\text { embryos }\end{array}$ \\
\hline \multicolumn{9}{|l|}{ Alfa-radiation } \\
\hline Gamma-radiation & ** & & & ** & + & 0 & - & \\
\hline Neutron-radiation & & & & ** & & 0 & & \\
\hline \multicolumn{9}{|l|}{$\begin{array}{l}\text { Hypomagnetic } \\
\text { field }\end{array}$} \\
\hline $\begin{array}{c}\text { Alfa-radiation + hypomagnetic } \\
\text { field }\end{array}$ & & & + & & & & & \\
\hline $\begin{array}{c}\text { Gamma- radiation + hypomagnetic } \\
\text { field }\end{array}$ & & & ++ & & & & & \\
\hline Gamma-and neutron-radiation & & - & & & & & & ${ }^{*}$ \\
\hline
\end{tabular}

Note: ${ }^{+}$positive effect; ${ }^{-}$negative effect; ${ }^{0}$ no effect; ${ }^{*}$ morphological deviations; ${ }^{* *}$ radiation hormezis.

"MIR" from the surfaces of construction materials were picked out some micromycetes with the series of morphological alterations [10].

Numerous of investigations $[11,12]$ is showed the immediate influence of ionized and non-ionized electromagnetic emanation on water molecules. Besides it is existed the dependence of water medium state from the physic environment [12]. For the investigation of the possibility of the water medium itself "accept" the radiation influence and induce the receiving "informational" alterations as the result of that effect on the bioobjects were studied the effect of low doses of hard $\gamma$ - $\left(\gamma\right.$, PAD $0.3 \mathrm{mGy} / \mathrm{day}$, the source ${ }^{137} \mathrm{Cs}$, the time of exposition 35 days) and neutron $\left(\mathrm{n}^{\circ}, 0.1-1.5 \mathrm{n}^{\circ} / \mathrm{sm}^{2} \cdot \mathrm{c}\right.$, the source $\mathrm{Pu}\left(\alpha, \mathrm{n}^{\circ}\right) \mathrm{Be}, \mathrm{PAD}-0.044 \mathrm{mGy} / \mathrm{day}$, the time of exposition 35 days) emanations on the micromycete development under nutritious medium. It was established the increasing of mushroom biomass under $\gamma$-irradiation that corresponds to a radiation hormezis [10]. As it is known via the supporting cell into the conidium-bearer join some nutritious things, moreover, the supporting cell is sensitive to the alterations occurring in the substrate. There were some deviations in morphology of supporting cell in Aspergillus niger which as a rule divided and increased into two and more times in sizes were marked under the $\gamma$-neutron radiation. Another deviation in the development of Aspergillus niger were numerous head falls in micromycetes under the $\gamma$-neutron radiation that corresponds to the answer of mushrooms for the effect of unfavorable factor for this culture. Consequently the $\gamma$-neutron radiation even such low doses (dose from $\gamma$ compiled $56 \mathrm{mrad}$, stream $\mathrm{n}^{\circ}=2 \times 10^{6} \mathrm{n}^{\circ} / \mathrm{sm}^{2}$ ) flight strains perceived as a factor of deterioration of the environment for them [6].

\section{SPIROSTOMUM AMBIGUUM EHRBG}

About the state of water also umpired for the alteration of spontaneous motion activity (SMA) of eyelash infusorium-spirostoms (Spirostomum ambiguum Ehbg.) accommodated both in the samples of water processing by radiation in cell (model) and in the samples are situated into the same room for the distance of $10 \mathrm{~m}$ from the irradiater (control). Spirostomes accommodated into the appointed samples of water in $2 \mathrm{~h}$ after the finishing of radiation effect and produced the measures of SMA.

Then, the number of crossings (SMA) decreased practically in 2 times under the mixed $\gamma$-neutron radiation, i.e. the alteration of water medium state under the radiation led to the changing of SMA of spirostomes that testifies about that the determinate factor of the effect of $\gamma$-neutron radiation is the alteration of the state of water medium. On the other hand, one-cell organisms are the good indicators of subtle alterations in the environment. The changing of hydro-bionts behavior is the first answer of animals for the transformation of water properties. So, in the investigations of the effect of radiation processing of water on SMA of spirostomes Spirostomum ambiguum Ehrbg. showed that the preliminary processing of substrate (settled water-piped water) led to the marked alteration of SMA. Moreover, the changing of SMA, i.e. the decreasing or its increasing depended on the type of radiation.

\section{DRY SEEDS OF THE HIGHEST PLANTS}

In our previous work [13] were studied the effect of radiation influence of low doses of $\alpha$ - and $\beta$-radiation on the rising and germination energy of the redishes seeds 
of Mohovskyi sort and the development of germinating seeds of 4 types of the high plants (wheat, kidney beans of Mash, lentil, mustard white).

The currents in water immediate radiated by low doses (less $10 \mathrm{cGy}$ ) of $\alpha$-particles steadily decreased and under the effect of $\beta$-particles of close intensity-increased in comparison with control [13]. It was supposed that under the interaction of ionized radiation and molecular formations occurs the changing of the structures apparently type $\mathrm{H}_{3} \mathrm{O}^{+}, \mathrm{H}_{7} \mathrm{O}_{3}^{+}$determining conductive properties of water. Experimentally in the experiences by-stand we were confirmed the indirect effect of the changing water medium state of the sample on the water medium-sensor. Some experimental data were also received about biological properties of radiation processing water on which the experiences for the germination of high plants seeds were carried out.

Then, there is the same decreasing of germination energy (till 20\%) and rising seeds (till 15\%) under the effect of $\alpha$ - and $\beta$-particles in comparison with control was marked, i.e. low-intensive irradiation of water induces both direct and indirect changing its properties and biological characteristics of the high plant seeds. The different radio-sensitivity of plants depending on species belonging and the way of preliminary radiation of water was exposed. So, wheat germinating seeds were indifferent to the radiation by the stated particles. The intensity of growing characteristics increased till $25 \%-30 \%$ in lentil and kidney beans of Mash. On the contrary, in plants of mustard white was established the decreasing of speed development of germinating seeds till $30 \%-40 \%$ in comparison with control. The deepest changing both in positive and in negative side under the seed germination observed in water of preliminary irradiation by beta-emanation (2 kBk) [13].

\section{JAPANESE QUAIL EMBRYOS}

The explorations of embryonic and post-embryonic development of Japanese quail under the effect of cosmic flight (CF) factors carried out series of experiments in the period of 1990-1998 on orbital complex "MIR". The results of investigations showed the possibility in conditions of CF the development the embryo from the egg fertilizing on the Earth $[14,15]$. However, the different morphological disturbances were discovered in part of embryos developing in conditions of CF. It isn't excluded that for the embryonic development of organism on the ship's side of orbital station could provide the influence of chronic irradiation by cosmic ionized emanation. It is known that living organisms on the stage of early embryogenesis are most sensitive to the effect of any factors of outer medium including the radiation. Moreover, the sensitivity displays not in the decreasing of survival of individuals but in the deviation from morphological and functional norm of the development. For the estimation of the chronic radiation effect by low doses of $\gamma$ - and neutron irradiation on the development of embryos of Japanese quail its fixation on the different periods of incubation produced.

During the investigation of embryos [16] developing in the period of 14 days under the influence of $\gamma$ radiation was showed that from 60 accommodating for the incubation quail eggs 3 occurred unfertilized but from the leaving 57 eggs 23 embryos conformed to the period of development (at the moment of fixation they were alive), 31 embryos were died and only 5 nestlings hatched out. Visual inspection of all embryos and incubation waste (fixed in the definite periods of eggs incubation) didn't reveal any differences for the main parameters between the experience and control group of the experiment. Morpho-metric parameters of embryos undergoing by the irradiation were in norm. However, there are numerous dot haemorrhages on the body were discovered in 5 embryos from the experience group. The nestlings from the experience group hatching out at 18th day of incubation conformed to the control period of development and were viable. At the 1st day of postembryonic development in the nestlings were taken blood. Clinical analysis of the total blood picture in quails undergoing in the period of embryogenesis by low doses of chronic $\gamma$-radiation showed that the regular elements of its blood hadn't any deviation from norm. The egg-laying in female from the experience group which was the only one from 3 nestlings reaching the puberty age started practically at the same periods that in the females from control group (42nd and 40th days, accordingly). Thus, the chronic irradiation by low doses of $\gamma$-radiation power $0.15 \mathrm{cGy} /$ day doesn't induce any pathological changing in the organogenesis of quails leaving alive at the moment of the carrying out the investigations.

Under the effect of $\gamma$-neutron radiation in surface model laboratory conditions [16] in embryos developing during 14 days under the low doses of neutron radiation more than $50 \%$ embryos from the accommodated eggs for the incubation conformed to the periods of development. The exploration of embryos deriving from the incubator at 10th and 14th days of development was showed that the embryos of this age didn't conform to the period of vital fixation, they had a lag in the development at 1 - 2 days in comparison with control. The leaving after the fixation 24 quail eggs were put into the laboratory incubator from them hatched out 11 viable nestlings. In 7 embryos ( $12 \%$ from the accommodated fertilized eggs) were discovered morphological disturbances. The most number of anomaly in embryos was connected with the eye development (microphtalmia and 
anophtalmia), the extremity development (twist fingers, shortening of the lower extremities), beak (parrot beak, underdevelopment of the upper or lower beak plate). The number of anomaly in the development and types of these disturbances discovered in the embryos of Japanese quail developing under the effect of low doses of neutron irradiation coincide with the results received in the studying of the bird embryogenesis in conditions of CF.

The embryos carrying out the whole cycle of embryo development in conditions of $\mathrm{CF}$ had the same morphological disturbances as the embryos developing in the conditions of the effect of chronic neutron irradiation. It ought to remark that every one from the quail embryos developing in conditions of CF had several anomalies of development, so in whole they had more total number of development anomalies than the embryos developing under the neutron irradiation. In 9 embryos carrying out $2 / 3$ of embryo development under the chronic neutron irradiation discovered full-blood of organ vessel and numerous dot haemorrhages in the head and body (in 5 embryos). Age of died embryos having the anomalies in his development practically corresponds the first half of embryo development. The types of anomalies discovering in embryos in conditions of $\mathrm{CF}$ and under the chronic irradiation by low doses of neutron radiation are coincided. In this connection it can supposed that one of possible reason induced the anomalies of development in embryos of Japanese quail in conditions of $\mathrm{CF}$ can consider cosmic ionized radiation.

It is differed two types of death of cells living organisms: reproductive revealing in the process of cell division and inter-phase which isn't connecting with cell division but cell damage can reveal in series of morphological or functional disturbances [7]. The disturbances in the course of histogenesis and in the formational relations of embryo parts act the large role in the origin of organ pathology. However, not for all stages of the development embryos have the same sensitivity [3]. Appearance of organs normal for structure but reduced size or with morphological and functional disturbances can explain with the different expression of formational processes and its combination. Some embryo cells possess high capacity to the regulation and accelerated duplication that can assist to the development of compensated organ from the destroyed laying. The rise of organ disturbance can be induced by the effect of radiation not only in the moment when it carries out its formation but much earlier on the stage of cells-predecessors. For the valuable development of organism it is necessary absolute non-disturbance of the structures and functions that apparently doesn't get to supply if the initial cell material even insignificantly was damaged by radiation. Evidently it can explain by this fact the rise of anomalies in the development in the early stages of embryogenesis under the low doses of radiation [7]. Thus, the presence of anomalies in organ structure of embryos apparently is connected with cell damages and the disturbances of formation induced by chronic irradiation of low doses of ionized radiation both in the conditions of $\mathrm{CF}$ and in surface experiments imitated the radiation state in orbital cosmic station.

\section{MEZENCHIM STEM CELLS OF MICE BONE BRAIN}

It is discovered [17] the effect of low doses of continuous $\gamma$-radiation (source of radiation $\mathrm{Co}^{60}$, period of radiation 10 days, average daily power dose $1.5-2.0$ $\mathrm{mGy}$, summary dose $15 \mathrm{mGy}$ ) on the hemato-poetic and stroma ancestor cells of mice bone brain. The states of bone brain stroma estimated by the clone method in vitro for the number of mezenchim stem cells (MSC) forming the colony of fibro-blasts (CFU-F) and by the method of ectopic transplantation for the capacity of stroma to organize and build new hemato-poetic territories. In bone brain by the method of Till and Mac-Calloc estimated the content of hemato-poetic ancestor cells (CFU-C). We were exposed the phenomena of stimulating effect of low intensive $\gamma$-radiation on MSC (radiation hormezis). It revealed in the intensification of proliferative activity and increasing in $1.5-4.5$ times the numbers of CFU-F in bone brain. Regenerative capacity of bone brain stroma estimating by the method of ectopic transplantation for the size again creating hemato-poetic territory essentially increased (in 2 times). Under neutron irradiation the radiation hormezis observed only in dose $0.48 \mathrm{mGy}$. In difference of stroma cells hemato-poetic ancestor cells were non-sensitive to the used radiation doses: the alterations of the number CFU-C in S-phase and its content in bone brain didn't occur.

The receiving data can be used for the prognosis of consequences of radiation effect of low intensity on tissue-specific stem human cells under the work in cosmos, enterprises of atomic industry, submarines, in conditions of increased radiation pollution, under high-altitude and high-latitudinal flights and so on. Besides the phenomena of radiation hormezis discovers new perspectives in the investigation of mezenchim cells having some various localization of tissue. The activation of cells being capable to the migration and possessing histogenetic plasticity can represent a serious biomedical problem.

\section{BLOOD LYMPHOCYTES OF PILOTS AND COSMONAUTS}

In the present it is proved that the radiation in low doses $(\sim 0.2 \mathrm{~Gy})$ can induce the whole number of effects on all levels of biological organization-the expression of speci- 
fic genes [18], the damage of genetic apparatus $[19,20]$, non-stability of genome $[21,22]$, the adaptive answer and the increasing of radio-sensitivity [23]. On the organism level increases the risk of the origin of malignant tumors, the number of non-tumor diseases $[24,25]$.

Under the inspection of pilot group (17 men, pilots of civil aviation, the flight high $6000-17000 \mathrm{~m}$, the number of hours $56-3500 \mathrm{~h}$, getting dose radiation from 0.019 to $1.4 \mathrm{cGy}$ ) and cosmonauts (6 men, later $10-20$ years after the finishing of flights with the duration of staying in cosmos from 10 to 679 days, the doses of radiation from 0.14 to $13.29 \mathrm{cGy}$ ) by the method of DNA-comet was revealed the damaging of DNA which was higher than in control group (16 men in age less of 50). After the adding radiation load (by the irradiation of lymphocyties out of the organism in dose $1 \mathrm{~Gy}$ ) the sensitivity of blood DNA-lymphocyties to the irradiation in pilots was average reliable higher than in control group. The distribution of parameters in cosmonauts in comparison with control despite on the equal of average differs for the criteria Mann-Whitny and Kolmogorova-Smirnova that testifies about more large variety of parameters than in control. In cosmonauts was revealed a significant individual variety. So, in 1 man was especially high the reaction for the radiation, at the same time the lymphocytes of some cosmonauts were resistive to the adding radiation load. Apparently it can suppose the presence of an adaptive answer. These data show that the method of DNA-comet allows discover some individual peculiarities of blood lymphocytes, mark some people with the high degree of its DNA damage and especially some sensitive to the adding radiation load. It is thought that this parameter is a biological marker for the definition of the early molecular disturbances in organism.

By the method of metaphase analysis of chromosome aberrations in pilots and cosmonauts established the aberrations of chromatid type. After the radiation of lymphocytes in dose 0.5 Gy revealed that in average radiosensitivity in pilot group was higher than in control group, but in cosmonauts was marked distinct tendency to it increasing. In pilots and cosmonauts was discovered the reliable adaptive answer. The capacity to the adaptation (adaptive answer) is the mutual inducible reaction of cells and organisms for the influence of chemical and physical agents in low doses expressed in the increasing of resistance to the subsequent effect the same or other agents in high doses. The increasing of sensitivity (but not the increasing of resistance) testifies about some disturbances in the system of organism homeostasis [26]. It is supposed that evidently some individuals with high reaction DNA lymphocytes for the radiation should carry away to the risk group. The capacity to the adaptive answer testifies about non-disturbing homeostasis, about the resistance to the adding influences. The reaction for the flight conditions, for the occurred radiation effects during the flight it is necessary to estimate in each separate man. Biological markers are the damage of DNA, the level of the frequency of chromosome aberrations, the reaction for these parameters to the adding radiation load in the type of adaptive answer allows determine some disturbances which can be early parameters of risk origin of the different diseases including malignant tumors $[4,27,28]$.

\section{WATER}

The influence of surrounding surface space on some biological objects essentially changes that must inevitable accompany by the alteration of inner medium of the bioobjects which basis consists of water. It is well-known that the concentration of water molecules in cell exceed by a factor of a hundred-thousand than the concentration of protein and other molecules including into the cytoplasm content. Water is capable to reflect the effect of outer factors in its structure changing which accordingly occur immediate effect on the physiological processes in human organism [29-32].

Our previous investigation [33,34] was showed that water possesses unusual sensitivity to the smallest even marked revealing of solar activity. Moreover, water medium direct undergoes for the effect of gelio-physic factors. As a result it can change the structure of water as a matter of fact that acts an immediate effect on the physiological processes in living organisms. The influence of outer factors on water estimated by the method supposed by V. V. Tsetlin [33,34] in which the integral quantitative characteristic of water is the value of oxidative capacity caused with the electron activity in water molecules. The criteria of changing serves the value of electric current passing in two-electrode cells in which was used the electrodes made from inert materialsstainless steel and platinum. The effects of formation and reconstruction of double electrode layers on the borders of metallic surface and water lay in the base of processes taking to the changing of the currents in cells. The shifts of electric potentials reflect the changing of energy state of water molecules induced by electromagnetic background both nature and technogenius origin. Under the continuous round-the-clock monitoring of currents in water were revealed its daily and season variations that reflect in the difference of the temporal dependence of value and form both in the daily and the night period of day. The variety of current value were marked also under the different duration of daily phase and at the moment of passing of extreme points of Sun and Moon state on horizon. Analogous daily dynamics we were discovered in the different geographical points besides Moscow: in the Crimea and the North Caucasus. The reliable dif- 
ferences of water properties clear revealed at the moment of rising, culmination and sunset of Sun. The last, in our opinion, was induced by the tidal-tint mobility in the earth crust. Some season differences in the values of the extremes of studying parameter were discovered. In winter the maximum extreme was observed at the moment of Sun rising, and other-at the moment of sunset was weaker. In the period of spring-summer the correlation of its values occurred opposite: one extreme was usually low than other in $1.5-2$ times. In the cells with steel electrodes the index became minimum at the moment when Sun achieved the culmination. The values of extremes and its correlation also depended on duration of Sun staying on horizon. It is necessary to mark that the character of daily dynamics of water properties suffered from some changing during two-year observation. The results receiving at the moment of Sun eclipse serve as an example of the deviations of norm dynamics of water properties.

Significant gelio-physic alterations in surround space were the possible reason of discovered fluctuations. At the moment of cover maximum by Moon the sun disk the current deviations from usual daily course of curve were observed. Anomaly oscillations were observed yet two days. The carrying out continuous round-the clock monitoring of currents allowed find the whole number of periodic oscillations of water properties (with time in 13.7 and 3.1 days; 6.3 and 1.4 hours, 54.7 and 45 minutes, and also in more shot periods equal 18.8 and 2 minutes). With less reliability the current fluctuations fix also in the period of approximately 20 seconds.

In our primary idea the revealed daily oscillations of currents can be caused by the variations of energy of electro-magnetic field and evidently transparent of earth ionosphere [35]. The possible reason of discovered current changing lays in the alteration of electron density in ionosphere induced by the decreasing of flow ultraviolet radiation in night hours or at the moment of sun eclipse. It is naturally leads to the decreasing of the intensity of near-surface electro-magnetic background. It is known that modern techno-genius sources in part round-the-clock mobile connection bring a contribution in high level of electro-magnetic radiation. Apparently oscillating processes with short periods 8.18 minutes have the same nature that magnetic micro-pulsations forming because of interaction of magnetic field of solar wind with magnetosphere of Earth.

Thus, the foregoing testifies about the important role of water medium under the effect of natural factors. The effect of gelio-and geo-physic factors on water organisms occur due to the influence on its outer and inner water medium. According to G. Ling [36], the role of regular functions in organism fulfills the cell hydrate membrane consisting of strongly polarized water mole- cules and apparently our revealing changing properties of water influence on its permeability. We discovered seasonal, daily and more short-periodical variations of electric currents in water medium allow approach to the understanding of the essence of the effect of cosmophysic and geo-physic factors on bio-sphere. Synchronize of the rhythms can be the reflection of normal physiological state of water organism. These very strong disturbances are capable tell on the changing of amplitude rhythms of biochemical parameters and it desynchronize. The studying of biochemical nature of adaptations on the effect of gelio- and geo-physic factors and also the elucidation of reactions mechanism and the reason connection between them and cosmic factors are represented the perspective direction as far as "in the system of medium-organism from the last one is established the deep metabolic connections with regard to the medium factors" (V. I. Vernadskii) [37].

It is proposed the hypothesis by V. V. Tsetlin [38] which explains the mechanism of the effect of cosmic and geo-physic factors on bio-sphere and nature of the connection of solar activity with the daily, season, annual and other rhythms in living nature. In the basis of this idea lay some experimental facts testifying about that in water of geo-sphere and in water medium of living organisms constantly changes the state of electron membranes in water molecules that is induced by the effect of electromagnetic background of surround space on it. The natural component of background forms under the relaxation induced by the tidal-tint processes in blocks, stoves and other formations drawing up the earth crust. The geophysics mark the existence so-called virial oscillations of the inertia moments weakly connecting with each other the separate parts of lithosphere and atmosphere which also accompany with the electromagnetic radiation of width frequency and amplitude range. It is clear that in such source of the generation of electromagnetic radiation simultaneously rise the photons of different length waves with noticeably different penetrating capacity. For this reason the radiation exiting from the lithosphere or rising in the atmosphere has variable intensity depending on the content and physic state of the laying under rocks. Besides the value of energy exit of radiation can suddenly change under the earthquakes and other tectonic processes characterizing for those or other geological formations and rocks in the upper part of lithosphere. For that to understand, what role does the changing of state of electron membranes of water molecules in living or non-living nature play it should intend that as it is known the degree of excitement of molecules determines the value of oxidative potential of water. In connection with that the concentration of water molecules in near-cellular medium and into the cells exceed by several digits the concentration of other 
reagents the metabolism processes into the organisms in part hydrolysis majority determinate by the reactive capacity of water. So, the speed of biochemical reactions and transport of protein and small organic molecules determine not only with membrane potentials but the state of water medium. Thus, in our opinion observed phenomena of changing of oxidative potential of water induced by the electromagnetic radiation of surrounding space can explain and "pour the light" on the nature of cosmic processes influences on biosphere of Earth, discovering by A. L. Chizhevskii and other investigations.

\section{REFERENCES}

[1] Vorontsov, I.V., Zhiljaev, E.G., Karpov, V.N. and Ushakov, I.B. (2002) Low radiation effects and human health. Voronezhskii State University, Moscow.

[2] Grigoriev, Yu. G., Popov, V.I., Shafirkin, A.V., et al. (1986) Somatic effects of chronic gamma-irradiation. Atomizdat, Moscow.

[3] Kuzin, A.M. (1995) The ideas of radiation hormezis in atom century. Nauka, Moscow.

[4] Pelevina, I.I., Aleschenko, A.V., Gotlib, V.Ya., et al. (2005) Reaction of blood lymphocytes of individual with somatic diseases on the radiation effect in low doses. Journal of Radiation Biology, 45, 412-415.

[5] Luckey, T.D. (1980) Hormesis with ionizing radiation. CRC Press, Boca Roton.

[6] Tsetlin, V.V., Zenin, S.V., Golovkina, T.V., et al. (2003) Role of water medium in the mechanism of the effect of super-low doses of ionized radiation. Journal of Biomedical Technologies and Radio-Electronic, 12, 20-25.

[7] Graevsky, E.L., Detlaf, T.A. and Mednikov, B.M. (1977) Regularities of individual animal development and control of ontogenesis processes. In: Graevskiy, E.L., Ed., Problems of the Biology Development. Environment and the Developing Organism. TSHA, Moscow, 91-125.

[8] Tsetlin, V.V., Bondarenko, V.A., Viktorov, A.N., et al. (2002) Variations of radiation situation and the development of microbial association on OC "MIR" depend on solar activity. Atlas of the temporal variations of nature, anthropogenic and social processes, Ianus-K, Moscow, 3, 556-560.

[9] Tsetlin, V.V., Bondarenko, V.A., Deshevaya, E.A., et al. (2001) The results of the investigations of the effect of radiation conditions on the environment of orbit station and the perspectives of using it for the decision of earthly ecological tasks. Proceedings of the Scientific Readings Devoted to the 40th Annual of the First Man Flight in Cosmos, Moscow, 12 April 2001, 16-18.

[10] Tsetlin, V.V. and Deshevaya, E.A. (2003) The effect of chronic irradiation by low doses of cosmic ionized radiation on the character of microbial association forming in the environment of orbit stations. Journal of Radiation Biology and. Radioecology, 43, 172-175.

[11] Ageev, I.M. and Shishkin, G.G. (2001) Correlation of solar activity with the electro-conductivity of water. Jour- nal of Biophysics, 46, 829-832.

[12] Mendeleev, D.I. (1994) Secret thoughts. Nauka, Moscow.

[13] Tsetlin, V.V., Levinskich, M.A., Nefedova, E.L. et al. (2008) Effect of low doses of ionized radiation on water substrate and the development of high plant seeds. Journal of Avia-Cosmic and Ecological Medicine, 3, 18-22.

[14] Meleshko, G.I., Shepelev, E. Ya., Gurieva, T.S., et al. (1991) Embrional development of birds under the conditions of weightnessless. Journal of Cosmic Biology and Avia-Cosmic Medicine, 1, 37-39.

[15] Gurieva, T.S., Dadasheva, O.A., Meleshko, G.I., et al. (1993) Gual embrional development under the conditions of weightlessness. Acta Veterinaria Brno, 62, 25-31. doi:10.2754/avb199362suppl60025

[16] Gurieva, T.S., Dadasheva, O.A., Tsetlin, V.V., et al. (2007) Effect of chronic irradiation by low doses of ionized radiation on embrional development of Japanese quail. Journal of Avia-Cosmic and Ecological Medicine, 1, 20-24.

[17] Domaratskaia, E.I., Starostin, V.I., Tsetlin, V.V., et al. (2003) Effect of 10-daily $\gamma$-radiation in low doses on mice bone brain cells. Journal of Radiation Biology and. Radioecology, 43, 213-215.

[18] Cucinotta, F.A., Wu, H., Shavers, M.R. and Geworge, K. (2003) Radiation dosimetry and biophysical models of space radiation effects. Gravitational and Space Biology Bulletin, 16, 11-18.

[19] Shevchenko, V.A. (1997) Integrative estimation of genetic consequences of the effect of ionized radiations. Journal of Radiation Biology and Radioecology, 37, 569576.

[20] Snyder, A.R. and Morgan, W.F. (2004) Radiation-induced chromosomal instability and gene expression profiling: Searching for clues to initiation and perpetuation. Mutation Research, 568, 89-96. doi:10.1016/j.mrfmmm.2004.06.048

[21] Mazuric, V.K. and Michailov, V.F. (2001) Radiationinduced instability of genome: Phenomena, molecular mechanisms, pathogenetic significant. Journal of Radiation Biology and Radioecology, 41, 272-289.

[22] Sevan'kaev, A.V., Michailova, G.F., Potetnya, O.I., et al. (2005) Results of dynamic cytogenetic observation for the children and teenagers living on the territories of radiation pollution after Chernobyl catastrophe. Journal of Radia- tion Biology and Radioecology, 45, 5-15.

[23] Morgan, W.F. (2003) Is there a common mechanism underlying genomic instability, bystander effects and other non-targeted effects of exposure to ionizing radiation? Oncogene, 22, 7094-7099. doi:10.1038/sj.onc.1206992

[24] Pelevina, I.I., Afanas'ev, G.G., Aleschenko, A.V., et al. (1999) Radio-induced adaptive answer in children and effect on it some outer and inner factors. Journal of Radiation Biology and Radioecology, 39, 106-112.

[25] Preston, D.L., Shimizu, Y., Pierce, D.A., et al. (2003) Studies of mortality of atomic bomb survivors. Report 13: Solid cancer and non-cancer disease mortality: 19501997. Radiation Research, 160, 381-407. 
doi:10.1667/RR3049

[26] Ivanov, V.K., Maksutov, M.A., Chekin, S. Yu., et al. (2005) Risk of cerebro-vascular diseases among the liquidators of the ChAES catastrophe. Journal of Radiation Biology and Radioecology, 45, 261-270.

[27] Pelevina, I.I., Antoschina, M.M., Bondarenko, V.A., et al. (2007) Individual cytogenetic and molecular-biological peculiarities of blood lymphocytes of pilots and cosmonauts. Journal of Radia- tion Biology and Radioecology, 47, 141-150.

[28] Ushakov, I.B., Zuev, I.G., Abramov, M.M., et al. (2001) Radiation risk in aviation flights. Voronezhskii State University, Moscow.

[29] Zenin, S.V. (1995) Mechanism of the effect of superlow doses via the alteration of informational system of water. Proceedings of the 2nd International Symposium: Mechanisms of the Effect of Superlow Doses, Moscow, 23-26 May 1995, 94-95.

[30] Zenin, S.V. (1997) Water medium as information matrix of biological processes. The 1st International Symposium on Fundamental Sciences and Alternative Medicine, Puschino, 1997, 12-13.

[31] Zenin, S.V. (1999) Complex-formation acetonitril and metyl alcohol with water. Journal of Physic Chemistry, 73, 835-839.
[32] Zilov,V.G., Cudakov, K.V. and Epshtein, O.I. (2000) Elements of informational biology and medicine. Meditsina, Moscow.

[33] Tsetlin, V.V. (2010) Studies into water reactions to variations of cosmophysical and geophysical factors of the environment. Journal of Avia-Cosmic and Ecological Medicine, 6, 26-31.

[34] Tsetlin, V.V. and Fineshtein, G.S. (2012) About the effect of cosmophysic, geophysic and radiation factors on the electro-physic and biological water properties. Journal of Metaphysics, 2, 81-99.

[35] Tsetlin, V.V., Artamonov, A.A., Bondarenko, V.A. and Fedotova, I.V. (2008) About temporal currents variations of water conductivity in electrochemical cell. Journal of Solar-Earth Physics, 2, 361-363.

[36] Ling, G.N. (2008) Life at the cell and below-cell level. The hidden history of a fundamental revolution in biology. Pacific Press, New York.

[37] Vernadskii, V.I. (2003) Biosphere in cosmos. Iris-Press, Moscow.

[38] Tsetlin, V.V., Zenin, S.V. and Lebedeva, N.E. (2005) Mechanism of the effect of superlow doses of ionized radiation on water medium. Journal of Biomedical Technology and Radioelectronics, 6, 53-57. 